\title{
@@- OPENACCESS EMERGENCE OF SUBSPECIALTIES IN ORTHOPAEDICS: THE ROAD FORWARD IN KHYBER PAKHTUNKHWA - PAKISTAN
}

\author{
Zeeshan Khan ${ }^{1 \times}$, Muhammad Arif Khan ${ }^{1,2}$
}

${ }^{1}$ Department of Orthopedics, Hayatabad Medical Complex Peshawar - Pakistan.

${ }^{2}$ Postgraduate Medical Institute, Peshawar - Pakistan.

Address for correspondence: Zeeshan Khan

Department of Orthopedics, Hayatabad Medical Complex Peshawar - Pakistan.

E-mail:

zeek1978@yahoo.co.uk

This article may be cited as Khan Z, Khan MA. Emergence of subspecialties in orthopaedics: The road forward in Khyber Pakhtunkhwa - Pakistan. J Postgrad Med Inst. 2021; 35(1): 1-2. https://doi.org/10.54079/ jpmi.2021.35.1.2975
Orthopaedics, in its evolutionary and developmental stages, focussed more on the generality of the specialty, specifically on fractures, dislocations, paediatric deformities, and infection. However, this has substantially changed lately and like all other specialties, the role of sub specialty training and practice has taken this field by a storm. Orthopaedic surgeons now can subspecialise in complex trauma management including pelvis and acetabular fractures; limb deformity correction; paediatrics; arthroplasty of both upper and lower limb; spine surgery; hand and upper limb including brachial plexus injuries; foot and ankle surgery; sports injuries surgery; and musculoskeletal oncology. Since the emergence of subspecialty trained orthopaedic surgeons, the proportion of practicing general orthopaedic surgeons has decreased from $44 \%$ to almost $28 \%$ in less than 2 decades (1990-2006) in the US alone. ${ }^{1}$ This has led to a further interest in seeking post residency fellowships, with around $90 \%$ residents undertaking an additional 1 or 2 years of training in their field of interest. ${ }^{2}$

A study published in 2003 recommends against fragmenting a specialty in many sub-specialties, arguing that the need for additional training following successful completion of a training program is non-evidential, thereby emphasizing on the importance of general orthopaedics for practice and structuring dedicated programs for sub-specialization. ${ }^{3}$ Contrastively, a study on job advertisements in the US medical market between 1984 and 2009 shows a statistically significant trend towards seeking orthopaedics sub-specialists as an essential criterion for recruitment, which highlights its demand and value. ${ }^{4}$ Hence, the completion of a structured general orthopaedics residency is suggested followed by subspecialisation in a further structured and accredited training programme.

In Pakistan, orthopaedics is one of the fastest evolving specialties and it has been trying to keep up with the modern trends in the world. Historically, it was practiced by some orthopaedic surgeons, who would share their operating theatre with general surgeons, the idea of which is highly impractical in the fast-paced world of today. Within the last decade, the demand for the general orthopaedic surgeons is slowly declining in hospitals in the urban community, mainly because of the increasing popularity of subspecialty training and fellowships after completion of residency program, which has led to an intense competition for the available training slots in accredited subspecialty fellowships. At the moment, Pakistan orthopaedic Association offers one-year subspecialty training in 7 disciplines: Trauma, Spine, Arthroplasty, Paediatrics, Hand Surgery, Musculoskeletal Oncology, and Sports injury surgery. None of these fellowships are currently approved by any accreditation bodies such as Pakistan Medical Council (PMC) and College of Physicians and Surgeons of Pakistan (CPSP). However, Postgraduate Medical Institute (PGMI) of Peshawar in liaison with Khyber Medical University is starting one such advanced training fellowship program in foot and ankle surgery, which will be accredited by PMC. It is also pertinent to note that there are no accredited foot and ankle surgeons in the country and no dedicated foot and ankle surgery training program exist and these injuries are currently dealt with in their generality by most practicing orthopaedic surgeons.

There are around 250 orthopaedic surgeons practicing in Khyber Pakhtunkhwa (KP) province. The general trend of practicing subspecialties within the country is mirrored in KP and all major urban hospitals in KP have employed or seek to employ subspecialists. This puts further pressure on the young aspiring surgeons who seek employment in hospitals or wish to practice independently. Thus, it becomes even more important for the job seekers to keep themselves abreast of the current job market and for the government to have a health service intelligence network to estimate the need of relevant physicians in each specialty in the coming years, which among others will particularly help the prospective trainees make informed decisions regarding their training.

According to the figures of PGMI Peshawar, which is also the sole conductor of residency programmes in KP, 71 residents had completed their fellowship (FCPS) training in Orthopaedics from 2012 to 2020. However, the number of residents currently undergoing training is 251 , who will be out in the job market in the coming few years. This may lead to more than $250 \%$ increase in the number of general orthopaedic surgeons as compared to the previous years. If the current trend 
continues, very soon the already scarce job market will be saturated with general orthopaedic surgeons. It is therefore a pressing priority, that CPSP and universities start PMC accredited subspecialty fellowships, providing an opportunity to general orthopaedic surgeons to train in subspecialties, thus raising the overall standard of patient care in orthopaedic surgery and making orthopaedic surgeons more employable. Also, with an increased emphasis now on swift outcomes and explicit expertise, the practice of subspecialty is likely to further expand and an interesting evolution in the specialty of Orthopaedics over the next decade is expected. The following suggestions are pertinent, in this context;

1. Development of a national or provincial health surveillance network to estimate the availability, demand and supply of physicians in different specialties.

2. The employment of this network to pave way for estimating the number of training slots needed in every specialty and help guide the deaneries or post graduate medical institutes to do the same. These figures will help young physicians/surgeons make informed decisions based on their preferences and job market.

3. Orthopaedic surgeons wishing to practice in tertiary care specialty hospitals have to subspecialise or super specialise in order to stand any chance of securing a job either in Government or independent sector.

4. Setting up of practices in routine care settings, either independent or group, offering general orthopaedic services and a sub specialty care feasible for, and in demand in the local hospital and population.

5. The aspiring surgeons must be mindful of the validity of a fellowship and of the fact that a visiting fellowship/observation of 1-3 months only means a trial for a fellowship beginner and should not be confused with a structured fellowship program.

\section{REFERENCES}

1. Gaskill T, Cook C, Nunley J, Mather RC. The financial impact of orthopaedic fellowship training. J Bone Joint Surg Am. 2009; 91(7):1814-21. https://doi:org/ 10.2106/JBJS.H.01139.

2. Salsberg ES, Grover A, Simon MA, Frick SL, Kuremsky MA, Goodman DC. An AOA critical issue. Future physician workforce requirements: implications for orthopaedic surgery education. J Bone Joint Surg Am. 2008; 90(5):1143-59. https:// doi:org/10.2106/JBJS.H.01139

3. Sarmiento A. Subspecialization in orthopaedics. Has it been all for the better? J Bone Joint Surg Am. 2003; 85(2):36973. https://doi:org/ 10.2106/00004623200302000-00028

4. Morrell NT, Mercer DM, Moneim MS. Trends in the orthopedic job market and the importance of fellowship subspecialty training. Orthopedics. 2012;35(4):e555-60. https://doi:0 rg/10.3928/01477447-20120327-13
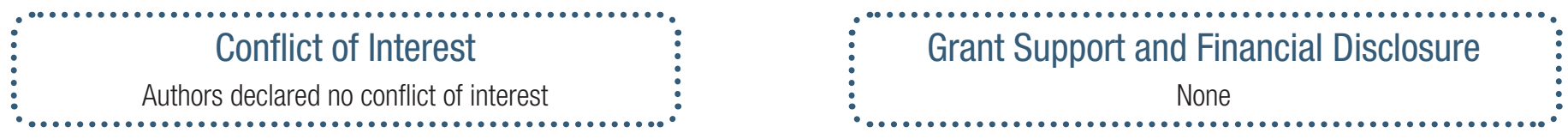